\title{
Magnetic Field and Temperature Dependencies Shed Light on the Recombination Kinetics of a Transition Metal Donor/ Acceptor System
}

\author{
T. von Feilitzsch, ${ }^{\dagger}$ P. Härter, ${ }^{\dagger}$ O. Schiemann,${ }^{\dagger}$ M. E. Michel-Beyerle $,^{*}, \dagger$ \\ U. E. Steiner, ${ }^{\ddagger}$ and P. Gilch*^§ \\ Contribution from the Department Chemie, Technische Universität München, \\ Lichtenbergstrasse 4, 85748 Garching, Germany, Fakultät für Chemie, Universität Konstanz, \\ 78457 Konstanz, Germany, and Department für Physik, Ludwig-Maximilians-Universität \\ München, Oettingenstrasse 67, 80538 München, Germany \\ E-mail: michel-beyerle@ch.tum.de; peter.gilch@physik.uni-muenchen.de
}

\begin{abstract}
The radical pair recombination of an intramolecular electron-transfer system containing a transition metal moiety has been addressed by femtosecond spectroscopy. The radical pair is formed by ultrafast electron transfer (90 fs) from a ferrocene residue to a photoexcited Nile blue moiety. Its recombination proceeds on the picosecond time scale in a multiexponential fashion. The kinetic pattern is a manifestation of spin processes competing with electron transfer. Magnetic field effects on these kinetics allow one to disentangle the two contr butions. Their temperature dependencies yield the activation parameters of the two processes. The discussion focuses on the mechanism of electron spin relaxation. Strong evidence for the Orbach/Kivelson mechanism will be given.
\end{abstract}

\section{Introduction}

Electron-transfer (ET) processes of transition metal compounds ${ }^{1}$ are involved in such diverse phenomena as respiration, ${ }^{2}$ corrosion, and catalysis. ${ }^{3}$ This has motivated numerous experimental studies aiming at the various factors that may influence the ET kinetics of such compounds. As for ET systems in general, the energetics and the distance between the donor (D) and acceptor (A) components are cardinal quantities determining the kinetics of the system. ${ }^{4-7}$ Spin processes may be a further factor influencing ET kinetics. In contrast to hyperfine induced spin conversion prevailing in organic systems $(>1 \mathrm{~ns}),{ }^{8-13}$ in compounds containing transition metals, spin processes can have an impact on picosecond ET dynamics, as shown in an exemplary way for oxazine $1 /$ ferrocene. ${ }^{14-16}$ These ultrafast spin

\footnotetext{
$\dagger$ Technische Universität München.

$\doteqdot$ Universität Konstanz

$\S$ Ludwig-Maximilians-Universität München.

(1) Astruc, D Electron Transfer and Radical Processes in Transition-Metal Chemistry; VCH: Weinheim, 1995

(2) Trautwein, A X Bioinorganic Chemistry; Wiley-VCH: Weinheim, 1997

(3) Bersuker, I B Electronic Structures and Properties of Transition Metal Compounds; Wiley: New York, 1996

(4) Marcus, R A J. Chem. Phys. 1956, 24, 966

(5) Marcus, R A ; Sutin, N Biochim. Biophys. Acta 1985, 811, 265

(6) Jortner, J J. Chem. Phys. 1976, 64, 4860

(7) Bixon, M ; Jortner, J Electron Transfer: From isolated molecules to biomolecules; Wiley: New York, 1999

(8) Michel-Beyerle, M E ; Haberkorn, R ; Bube, W ; Steffens, E ; Schröder H ; Neusser, H J ; Schlag, E W Chem. Phys. Lett. 1976, 254, 384

(9) Schulten, K ; Staerk, H ; Weller, A ; Werner, H J ; Nickel, B Z. Phys. Chem. Frankfurt 1976, 101, 371

(10) Hoff, A J Q. Rev. Biophys. 1981, 14, 599

(11) Salikhov, K M ; Molin, Y N ; Sagdeev, R Z; Buchachenko, A L Spin Polarization and Magnetic Field Effects in Radical Reactions; Elsevier: Amsterdam, 1984

(12) Volk, M ; Aumeier, G ; Langenbacher, T ; Feick, R ; Ogrodnik, A ; MichelBeyerle, M E J. Phys. Chem. B 1998, 102, 735

(13) Steiner, U E; Ulrich, T Chem. Rev. 1989, 89, 51
}

processes may play a role in biological systems involving radical pair (RP)-type intermediates, where one part is a paramagnetic metal ion, as in a vitamin $\mathrm{B}_{12}$ analogue. ${ }^{17,18}$

Possible effects of a transition metal moiety on RP recombination kinetics are illustrated in the following (Figure 1). As a first step, let us consider a photoinduced ET between an organic acceptor cation $\mathrm{A}^{+}$and an organic donor $\mathrm{D}$, as shown in Figure 1a. Donor and acceptor are supposed to have closedshell, i.e., singlet-phase ground states. As the photoexcitation of $\mathrm{A}$ is a spin-conserving process, the system ${ }^{1}\left(\mathrm{D} \mathrm{A}^{+*}\right)$ is singlet-phased. Assuming favorable energetics, $A^{+*}$ accepts an electron from $D$ and the radical pair $(\mathrm{RP}){ }^{1}\left(\mathrm{D}^{+\bullet} \mathrm{A}^{\bullet}\right)$ is formed.

A large distance between the two unpaired electrons results in a small exchange interaction $J$, i.e., the singlet and the triplet states of the RP are quasi iso-energetic. Still, starting from a singlet excited state, only the singlet RP state ${ }^{1}\left(\mathrm{D}^{+\bullet} \mathrm{A}^{\bullet}\right)$ will be populated in the course of the ET process since, during elementary processes, spin multiplicity is conserved. As the RP recombination (or backward ET, BET) originates from a single (and singlet) state ${ }^{1}\left(\mathrm{D}^{+\bullet} \mathrm{A}^{\bullet}\right)$, the decay of the RP population is single-exponential. The triplet $\mathrm{RP}^{3}\left(\mathrm{D}^{+\bullet} \mathrm{A}^{\bullet}\right)$ comes into play if interactions of the electron spins and other magnetic moments drive transitions between the spin states. Such interactions are effective only if their magnitude (in angular frequency units) is

(14) Gilch, P ; Pöllinger-Dammer, F ; Steiner, U E; Michel-Beyerle, M E Chem. Phys. Lett. 1997, 275, 339

(15) Gilch, P ; Pöllinger-Dammer, F ; Musewald, C ; Michel-Beyerle, M E Steiner, U E Science 1998, 281, 982

(16) Steiner, U E; Gilch, P, High Magnetic Fields in Chemistry In High Magnetic Fields, Science and Technology; Herlach, F, Miura, N, Eds World Scientific: Hackensack, NJ, 2003; Vol 2, p 219

(17) Kräutler, B ; Stepanek, R Angew. Chem., Int. Ed. Engl. 1985, 24, 62 (18) Grissom, C B Chem. Rev. 1995, 95, 3 


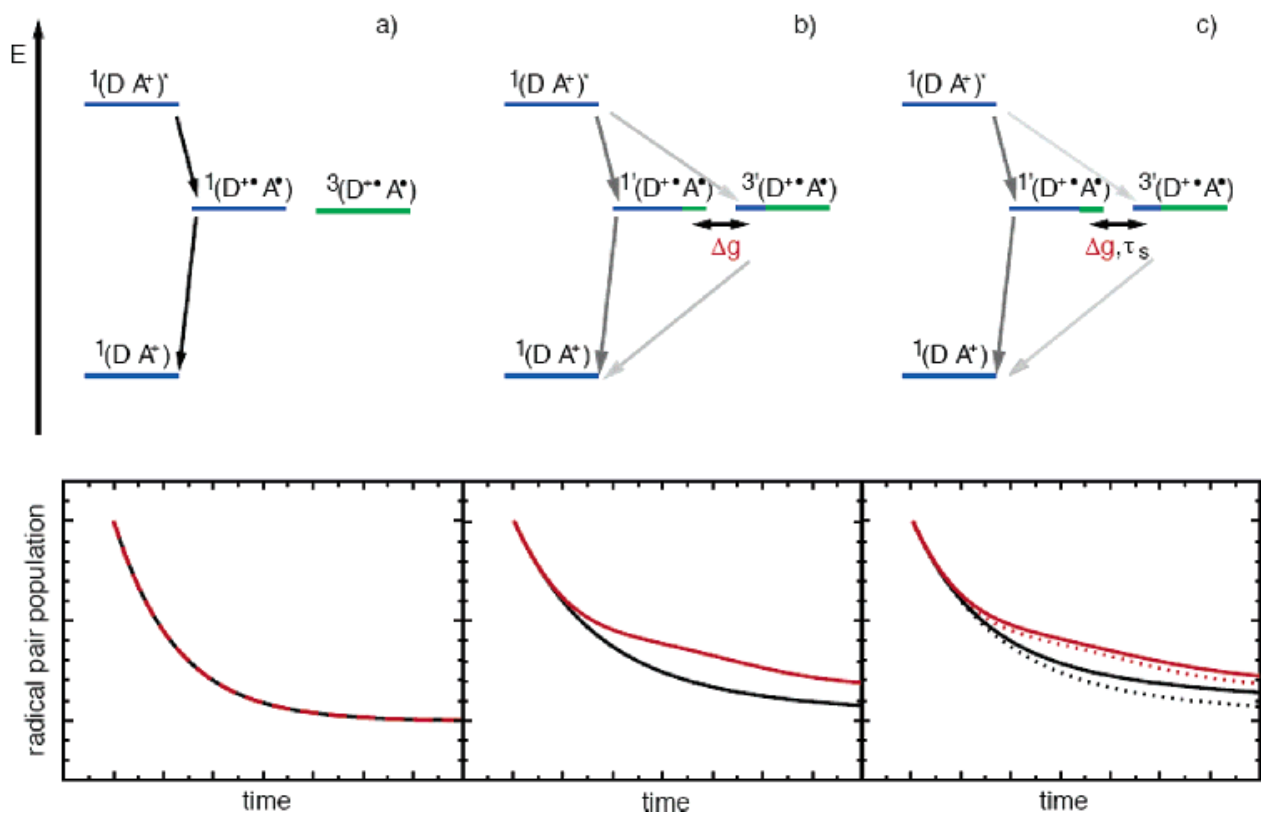

Figure 1. Illustration of spin effects in fast RP recombination (top) and their influence on the RP population in zero (black lines) and high (red lines) magnetic field (bottom). Primed ' labels for multiplicity refer to Kramers notation (see text, Model and Data Analysis section, for details). Singlet (triplet)phase states are marked in blue (green). Gray shadows correlate with the relative contribution of singlet spin character of states involved in forward or back ET. The numerical parameters used for simulating the time traces are close to those best describing the experiments reported here. (a) Organic RP: Starting from a singlet precursor ${ }^{1}\left(\mathrm{D} \mathrm{A}^{+}\right)^{*}$, only the singlet-phase RP state is populated and decays in single-exponential fashion. The triplet-phase RP states are not populated. (b) RP bearing a transition metal moiety (static effect, no spin relaxation): Spin-orbit coupling mixes the multiplicities; consequently, more than one RP state will be populated. As each state may recombine with a different rate, the RP decay kinetics are multiexponential. In an external magnetic field, transitions between the RP states due to the $\Delta g$-driven magnetic mixing lead to altered recombination kinetics. This effect can be used as a diagnostic tool for the kinetics in zero field. (c) RP comprising a transition metal moiety (fast spin relaxation): In addition to state mixing, spin-orbit coupling will also speed up relaxation-induced transitions among the RP states. Thereby, the RP kinetics can become even further involved. The field effect now is less pronounced. This damping is directly correlated with the relaxation time of the spin located at the transition metal. For better comparison, the time traces corresponding to scenario (b) are included as dotted lines.

comparable to or larger than the decay rate constant of the RP. In organic RPs the dominating interaction is the hyperfine interaction, with a typical ${ }^{19}$ magnitude of $10^{7}-10^{8} \mathrm{rad} \mathrm{s}^{-1}$. Thus, hyperfine interaction will only affect kinetics of organic RPs with lifetimes longer than several nanoseconds. ${ }^{8-13}$

A different situation may prevail for RPs with an unpaired electron located on a transition metal moiety. (We assume that the donor $\mathrm{D}$ contains the transition metal ion and is not photoexcited.) In transition metal ions, small ligand field splitting of partially filled d-orbitals in combination with large spin-orbit coupling constants results in strongly coupled spinorbit states. In other words, in such compounds the factorization of the electronic wave function in a spin and an orbital part often fails. For the kinetics of the RP, this has two consequences. Since the spin state of a "transition metal radical" cannot be described by a simple spin wave function $(\alpha$ or $\beta$ ), this is also not possible for a RP bearing such a radical. This RP will not have pure singlet or triplet wave functions but wave functions describing mixtures of the two multiplicities (see Figure 1b). Thus, even when starting from a purely singlet-phase excited state, RP states with a partial triplet character will be populatedwith concomitant consequences on the decay of the RP, resulting in non-single exponential kinetics. Furthermore, in strongly spin-orbit coupled systems, spin relaxation times can be very short (down to picoseconds). Spin relaxation then leads to incoherent transitions between the different RP states-again with consequences for the RP kinetics (Figure 1c).

(19) Ayscough, P B Electron Spin Resonance in Chemistry; Methuen \& Co Ltd : London, 1970
Experimental access to these spin effects involving transition metals can be gained via the modulation of the kinetics by static magnetic fields. ${ }^{13}$ An external magnetic field alters the transition rates between the RP states in a predictable way and allows conclusions to be drawn on these rates in a zero-field situation. In the present case, the field-induced transitions are caused by the $\Delta g$ mechanism. In spin-orbit coupled systems the $g$-factor of the unpaired electron deviates from that of the free electron, $g_{\mathrm{e}} \approx 2$, and is no longer isotropic. Thus, the electron spin located at the organic acceptor $\mathrm{A}^{\bullet}$ and the one located at the transition metal ion $\mathrm{D}^{+\bullet}$ precess with different angular frequencies. This results in a continuous rephasing of the RP states. The effect of this rephasing was first observed as a modulation of reaction yields in the photo-oxidation of ruthenium(II) complexes. ${ }^{20,21}$ Later, it was shown that the same approach can be used to analyze the photosensitized oxidation of ferrocene. ${ }^{15,22}$ In these experiments, effects on both the reaction yields ${ }^{22}$ and directly on the reaction kinetics ${ }^{15}$ were observed.

In the latter experiment, ${ }^{15}$ the organic dye oxazine 1 is excited by femtosecond laser pulses. The excited dye then accepts an electron from a ferrocene derivative (ethylferrocene) present in high concentration $(\sim 2 \mathrm{M})$. The forward ET (FET) process takes place on a time scale of $\sim 200 \mathrm{fs}$, forming a RP consisting of a ferricenium cation and the semireduced form of oxazine 1, both species solvated in ethylferrocene and acetonitrile $(\sim 1: 1 \mathrm{v}: \mathrm{v})$. This represents a situation similar to the ultrafast intermolecular

(20) Ferraudi, G ; Arguello, G A J. Phys. Chem. 1988, 92, 1846

(21) Wolff, H J ; Steiner, U E Z. Phys. Chem. N. F. 1990, 169, 147

(22) Gilch, P ; Linsenmann, M ; Haas, W; Steiner, U E Chem. Phys. Lett. 1996, 254, 384 

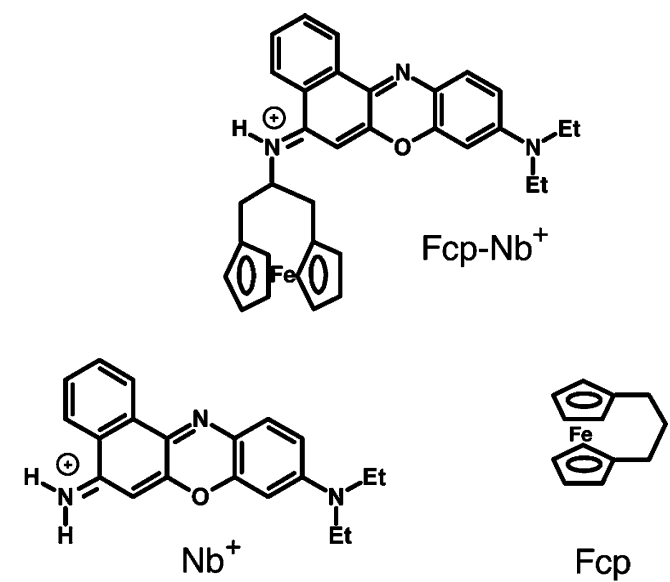

Figure 2. Structures of the intramolecular ET system $\mathrm{Fcp}-\mathrm{Nb}^{+}$and its constituents.

ET between solvent and solute ${ }^{23}$ reviewed in ref 24 . The RP recombines on the picosecond time scale in a multiexponential fashion. Applying magnetic fields of some teslas slows down the recombination. An analysis based on a stochastic Liouville equation approach (for details see below) shows that the multiexponential reaction kinetics are a direct consequence of the involvement of spin processes. Furthermore, the "unmasked" BET rate for the RP as well as the spin relaxation time of the ferricenium cation can be extracted with $k_{\mathrm{BET}}=(0.8 \mathrm{ps})^{-1}$ and $\tau_{\mathrm{S}}=6.5 \mathrm{ps}$. This underscores the above notion that in a transition metal D/A system, both BET and spin dynamics can occur on the picosecond time scale and may influence recombination dynamics significantly.

The intermolecular ET system described above prohibits temperature-dependent studies since the solution exhibits phase separation upon cooling. To overcome this limitation and to minimize the influence of diffusion, the intramolecular ET system $\mathrm{Fcp}-\mathrm{Nb}^{+}$has been synthesized, bearing a ferrocenophane (Fcp) and a Nile blue $\left(\mathrm{Nb}^{+}\right)$moiety (Figure 2). In a femtosecond transient absorption experiment aiming mainly at the kinetics of the RP formation, ${ }^{25}$ it was demonstrated that the ET kinetics of the intramolecular system are similar to those of the bimolecular system. In the present work, we will explore in detail the kinetics of the RP recombination in particular with respect to the interplay between ET and spin dynamics as well as their temperature dependence. This temperature dependence of the spin relaxation points to an Orbach/Kivelson-type relaxation mechanism involving an electronically excited Kramers doublet state.

\section{Experimental Results}

Selective photoexcitation of the $\mathrm{Nb}^{+}$moiety of $\mathrm{Fcp}-\mathrm{Nb}^{+}$is easily achieved by tuning the pump pulse to the absorption band centered at $642 \mathrm{~nm}$, which strongly resembles the one of the free $\mathrm{Nb}^{+}$dye (Figure 3), as the Fcp moiety does not absorb in this spectral region. The population of the photoexcited state ${ }^{1}\left(\mathrm{Fcp}-\mathrm{Nb}^{+*}\right)$ is followed by probing the stimulated emission at $700 \mathrm{~nm}$ and decays within the response time ( $\sim 200 \mathrm{fs})$ of the

(23) Kandori, H ; Kemnitz, K ; Yoshihara, K J. Phys. Chem. 1992, 96, 8042

(24) Yoshihara, K Ultrafast Intermolecular Electron Transfer in Solution In Electron Transfer: From isolated molecules to biomolecules; Jortner, J Bixon, M , Eds ; Wiley: New York, 1999; Vol 107

(25) Baigar, E; Gilch, P ; Zinth, W ; Stöckl, M ; Härter, P ; von Feilitzsch, T ; Michel-Beyerle, M Chem. Phys. Lett. 2002, 352, 176

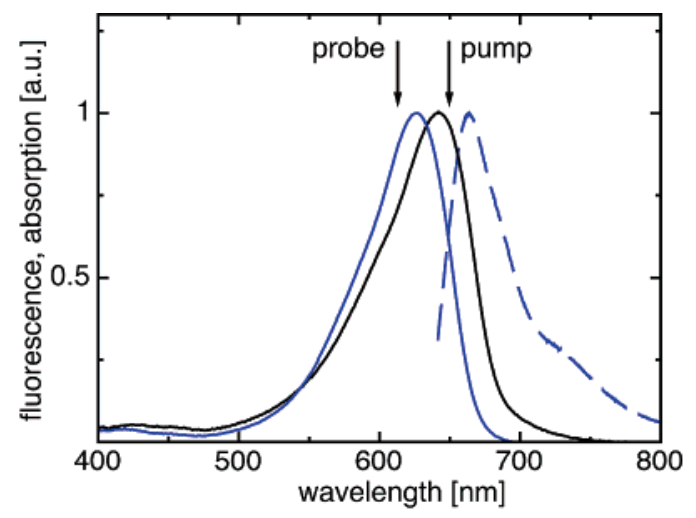

Figure 3. Absorption (blue line) and fluorescence (dotted line) spectra of $\mathrm{Nb}^{+}$and absorption spectrum of Fcp- $\mathrm{Nb}^{+}$(black line) in 2:1 ethanol: methanol $(\mathrm{v}: \mathrm{v})$. The arrows indicate the spectral positions of pump and probe beam for the femtosecond MFE experiments.

femtosecond setup (data not shown). This is completely in line with the results of the foregoing study ${ }^{25}$ in which with a setup with a higher time resolution of $\sim 15 \mathrm{fs}$ and an $S_{1}$ lifetime of $90 \mathrm{fs}$ was determined. The additional information obtained in the experiments here is that this lifetime stayed below the 200 fs response time in a temperature range between 295 and 170 $\mathrm{K}$. Furthermore, within the limit given by the response time, the $S_{1}$ lifetime is not affected by magnetic fields of up to $7 \mathrm{~T}$.

The rapid $S_{1}$ decay is assigned to an ET process from the (ground-state) Fcp moiety to the excited $\mathrm{Nb}^{+}$part forming a $\left(\mathrm{Fcp}^{+} \bullet-\mathrm{Nb}^{\bullet}\right) \mathrm{RP}^{25}$ The recombination of the radical pair, i.e., the BET process, repopulates the electronic ground states of the two moieties ${ }^{1}\left(\mathrm{Fcp}-\mathrm{Nb}^{+}\right)$and therefore is probed as a strong difference absorption signal at $610 \mathrm{~nm}$ ("ground-state bleaching"). Ground-state recovery (Figure 4, left column) is evidently much slower than the decay of stimulated emission, underscoring once again that, in $\mathrm{Fcp}-\mathrm{Nb}^{+}$, an intermediate state (namely the RP) is formed upon the decay of the excited state. The ${ }^{1}(\mathrm{Fcp}-$ $\mathrm{Nb}^{+}$) ground state recovers in a multiexponential pattern with characteristic times ranging from 1 to $100 \mathrm{ps}$. After $1 \mathrm{~ns}$ only a small offset is seen, most likely due to a signal contribution of a byproduct (see Experimental Section). Upon cooling to 170 $\mathrm{K}$, the characteristic times shift to slightly longer values, the recombination slows down.

At all temperatures, a strong magnetic field of $7 \mathrm{~T}$ affects the recombination kinetics. The common features of this effect are the following: around time zero the time traces at zero field and at $7 \mathrm{~T}$ are identical in magnitude and slope. In other words, the magnetic field does not affect the initial recombination rate given by the derivative of the time trace around time zero. From delay times of $\sim 1 \mathrm{ps}$ onward, the $7 \mathrm{~T}$ traces starts to deviate from the zero field traces. In high magnetic field the recombination of the RP is delayed. This effect is most prominent around 3-6 ps; toward longer times, the influence of the magnetic field becomes again marginal. Time traces at intermediate field strengths lie between the zero and $7 \mathrm{~T}$ traces (data not shown). The field effect is slightly sensitive to the temperature. The smallest effect is seen at $295 \mathrm{~K}$, and it is most pronounced at $170 \mathrm{~K}$.

The observed effects are caused by field-induced transitions between the spin states of the RP. The active part in the RP responsible for these transitions is the oxidized Fcp moiety 


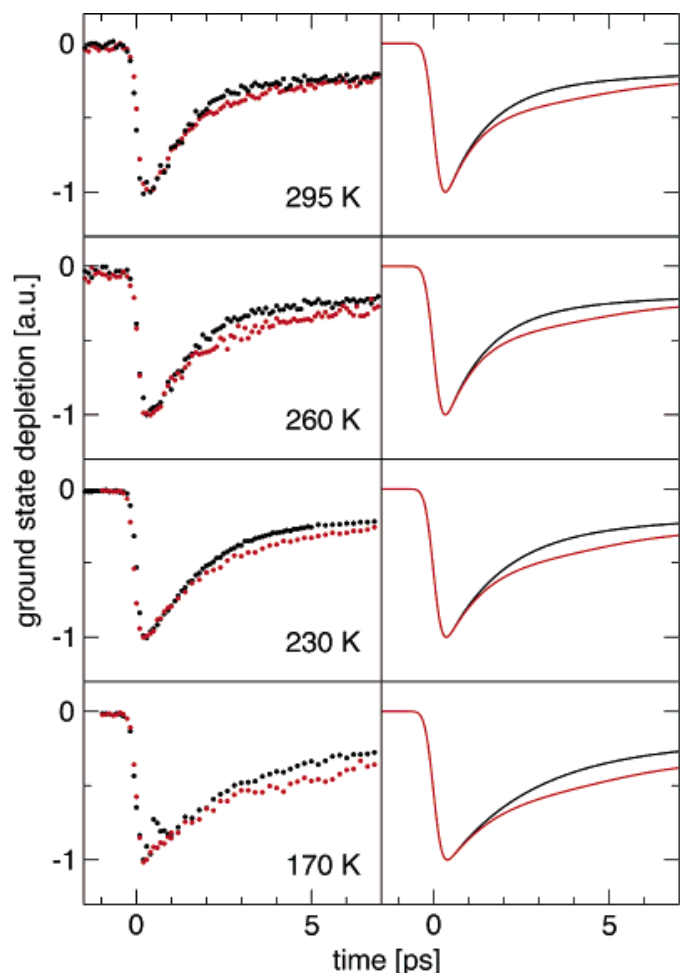

Figure 4. Ground-state recovery of $\mathrm{FcNb}^{+}$at $0 \mathrm{~T}$ (black lines) and $7 \mathrm{~T}$ (red lines). Sample temperatures are indicated. To compare these results with the illustration in Figure 1, the times traces have to be multiplied by -1 . Left: Measured time traces probed at $610 \mathrm{~nm}$ after excitation at 650 nm. Right: Simulation of the experimental time traces with the model described in text.

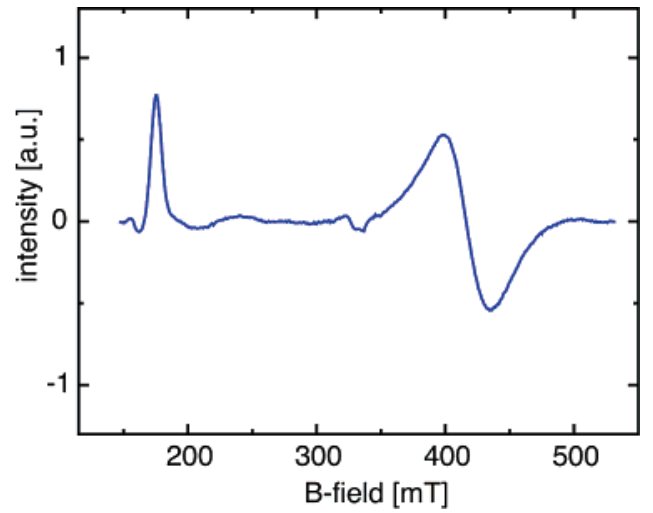

Figure 5. EPR spectrum of $\mathrm{Fcp}^{+\bullet}$ measured at $10 \mathrm{~K}$ at $9.42 \mathrm{GHz}$ in the usual differential representation. Conditions: $0.5 \mathrm{mM} \mathrm{Fc}, 0.5 \mathrm{mM} \mathrm{AgBF}_{4}$ in $\mathrm{MeOH}$.

$\left(\mathrm{Fcp}^{+\bullet}\right)$. As outlined in ref 26 and later on with emphasis on ferricenium cations in refs 15 and 22, relevant information on these transition can be derived from EPR spectroscopy. ${ }^{27}$ EPR spectra of ferricenium cations respond rather sensitively to distortions of the two cyclopentadienyl rings. ${ }^{28}$ In the bridged Fcp moiety, the two rings are strongly distorted by the $\mathrm{C}_{3}$ bracket. To quantify the effect of this distortion, the X-band EPR of $\mathrm{Fcp}^{+\bullet}$ was measured. Fcp was oxidized by addition of $\mathrm{AgBF}_{4}$ to a solution of this compound. The EPR spectrum (Figure 5) of $\mathrm{Fcp}^{+\bullet}$ is characteristic for a compound with large axial $g$-tensor anisotropy. The values of the $g$-tensor are $g_{\perp}=$

(26) Steiner, U E ; Bürssner, D Z. Phys. Chem. N. F. 1990, 169, 159

(27) Warren, K D Struct. Bonding 1976, 27, 45

(28) Ammeter, J H J. Magn. Reson. 1978, 30, 299
1.61 and $g_{\|}=3.86$, with the "parallel" value representing the situation of the magnetic field aligned normal to the cyclopentadienyl rings. We note that this anisotropy is smaller than the one reported ${ }^{29,30}$ for the ferricenium cation, with values of $g_{\perp}$ $=1.3$ and $g_{\|}=4.4$. The substitution of the ferrocenophane in $\mathrm{Fcp}-\mathrm{Nb}^{+}$should not influence the distortion of the cyclopentadienyl rings. Thus, the $g$-values of $\mathrm{Fcp}^{+\bullet}$ are expected to be valid for $\mathrm{Fcp}^{+} \cdot-\mathrm{Nb}^{\bullet}$, too.

\section{Model and Data Analysis}

The data analysis relies on a model ${ }^{26,31}$ which has been successfully employed to deal with the RP recombination of the intermolecular ET analogue to the present RP. ${ }^{15}$ We will briefly review this model and then demonstrate how crucial input parameters for the model can be extracted from the EPR spectrum and finally determine rate parameters by comparison of simulations based on this model with the experimental results. In the Introduction, we stated that the total spin is no longer a good quantum number for RPs bearing transition metal ions. Instead, it proves to be useful to treat the RP states as a combination of the spin wave function $(\alpha$ or $\beta$ ) of the organic radical moiety $\left(\mathrm{Nb}^{\circ}\right)$ with the lowest spin-orbit states of $\mathrm{Fcp}^{+} \cdot$ $\left(\alpha^{\prime}\right.$ or $\left.\beta^{\prime}\right)$. Also in the presence of spin-orbit coupling, a molecule or ion with one unpaired electron will retain a twofold degeneracy that is only lifted by magnetic fields (Kramer's doublet). Arranging the wave functions of the two unpaired electrons in the common fashion, i.e.

$$
\begin{gathered}
T_{+}{ }^{\prime}=\alpha \alpha^{\prime} \\
T_{-}{ }^{\prime}=\beta \beta^{\prime} \\
T_{0}{ }^{\prime}=\frac{1}{\sqrt{2}}\left(\alpha \beta^{\prime}+\beta \alpha^{\prime}\right) \\
S^{\prime}=\frac{1}{\sqrt{2}}\left(\alpha \beta^{\prime}-\beta \alpha^{\prime}\right)
\end{gathered}
$$

provides a set of basis functions for RP states. Note that this is a symbolic notation. In actual calculations, one has to work with Slater determinants to account for the indistinguishablity of the electrons. The priming of the symbols denotes that these effective spin functions are not spin functions of precisely defined multiplicities. Yet states denoted $T$ ' carry predominately triplet character, whereas the one denoted $S^{\prime}$ has predominately singlet character. The singlet character $p_{\mathrm{s}}$ of these states can be derived from EPR data as outlined below. Using these basis functions, the time evolution of the RP is described by the following stochastic Liouville equation:

$$
\frac{\mathrm{d} \rho_{\Omega}}{\mathrm{d} t}=-\frac{i 2 \pi}{h}\left[\hat{H}_{\Omega}, \rho_{\Omega}\right]_{-}+\hat{\hat{R}}_{\rho_{\Omega}}-\frac{1}{2}\left[\hat{K}, \rho_{\Omega}\right]_{+}
$$

Here, $\rho$ denotes the spin density matrix in the basis of the effective pair spin states specified above, its diagonal elements presenting the populations of the four RP states, and $\Omega$ symbolizes the orientation of a particular RP with respect to

(29) Prins, R Mol. Phys. 1970, 19, 603

(30) Materikova, R B ; Babin, V N ; Solodovnikov, S P ; Lyatifov, I R ; Petrovsky, P V; Fedin, E I Z. Naturforsch. (B) 1980, 35, 1415

(31) Bürssner, D ; Wolff, H J ; Steiner, U E Z. Phys. Chem. N. F. 1993, 254, 384 
the magnetic field. [... $]_{-,+}$stands for the commutator and anticommutator, respectively. The first term of this equation describes the coherent evolution of the RP states induced by the external field. This evolution is caused by the difference of the $g$-values of the two radicals ( $\Delta g$ mechanism). The Hamiltonian matrix $\hat{H}_{\Omega}$ depends only on the magnetic field, the $g$-values of the two radicals, and the orientation $\Omega{ }^{26}$ The orientation dependence is a manifestation of the anisotropic $g$-tensor of $\mathrm{Fcp}^{+\bullet}$. Trivially, this first term vanishes in zero field. The second term accounts for incoherent transitions among the $\mathrm{RP}$ states induced by spin relaxation. The relaxation drives the relative population of the four RP states toward equilibrium and destroys coherence among these states. The matrix elements of the relaxation super operator $\hat{\hat{R}}$ are determined solely by the spin relaxation time $\tau_{\mathrm{s}}$ of $\mathrm{Fcp}^{+\bullet} .22$ The longitudinal and transverse relaxation times are taken to be equal. ${ }^{31}$ The short relaxation time is a further manifestation of the large spinorbit coupling at the $\mathrm{Fcp}^{+\bullet}$ moiety. The relaxation of the spin located at the $\mathrm{Nb}^{\bullet}$ moiety does not have to be considered since the spin relaxation times of organic radicals are by several orders of magnitude too long to be of importance here. ${ }^{19}$ The last term describes the depletion of the RP states by recombination. The reaction operator $\hat{K}$ is a diagonal matrix; its diagonal elements are the recombination rate constants of the individual RP substates, given by the product of a rate constant $k_{\mathrm{BET}}$ and the singlet character $p_{\mathrm{s}}$. The rate constant $k_{\mathrm{BET}}$ characterizes the ET between a purely singlet-phase RP state and a singlet recombination product. Since in the present case all substates are more or less triplet-contaminated, the rate constant has to be reduced by the factor $p_{\mathrm{s}} \leq 1$.

Having outlined the formalism for the problem, we now have to find numerical parameters to perform the simulations. For the Hamiltonian $\hat{H}_{\Omega}$ this is straightforward. The parameters required are the $g$-tensor values of $\mathrm{Fcp}^{+\bullet}$ determined above and the $g$-value of the $\mathrm{Nb}^{\bullet}$ radical, which is taken to be $g_{\mathrm{e}} \approx 2$, as commonly found for organic radicals. RPs in solution are randomly distributed with respect to the magnetic field, and thus accounting for the orientation dependence requires taking an orientational average. The relaxation super operator is determined by one single parameter, namely the spin relaxation time $\tau_{\mathrm{s}}$. Its value will result from the simulation described below. This also applies for the ET rate constant $k_{\mathrm{BET}}$, one of the parameters of the reaction operator $\hat{K}$. The singlet characters $p_{\text {s }}$ can be derived independently from the $g$-tensor values of $\mathrm{Fcp}^{+}$. A previous analysis ${ }^{22}$ afforded the singlet characters as follows: $p_{\mathrm{s}}\left(T_{+}{ }^{\prime}, \mathrm{T}_{-}{ }^{\prime}\right)=0, p_{\mathrm{s}}\left(T_{0}{ }^{\prime}\right)=0.5-g_{\perp} / 4=0.1$, and $p_{\mathrm{s}}\left(S^{\prime}\right)$ $=0.5+g_{\perp} / 4=0.9$. The numerical values are based on $g_{\perp}=$ 1.61 as given above. The singlet character determines both the initial population and the depopulation rate of the RP states. The RP is formed by an ET process from a singlet precursor state; the RP states are thus populated according to their singlet character.

Altogether, only two parameters $\left(k_{\mathrm{BET}}\right.$ and $\left.\tau_{\mathrm{s}}\right)$ remain to be determined from the simulation. The stochastic Liouville equation was solved numerically, affording a time-dependent spin density matrix $\rho(t)$. The time interval in the numerical integration was set to $10 \mathrm{fs}$. For each time $t$, the trace of $\rho(t)$ was calculated, which is equal to the total population of the RP (sum over all RP states). It is this total population which is observed experimentally as the ground-state recovery signal.
Table 1. Rate Constants for Electron Transfer and Spin Relaxation Resulting from Simulating the Recombination Time Traces at Zero Field and at $7 \mathrm{~T}^{\mathrm{a}}$

\begin{tabular}{lccccc}
\hline & $295 \mathrm{~K}$ & $260 \mathrm{~K}$ & $230 \mathrm{~K}$ & $170 \mathrm{~K}$ & $E_{\mathrm{A}}$ \\
\hline$\left(k_{\mathrm{BET}}\right)^{-1}$ & $1.2 \mathrm{ps}$ & $1.2 \mathrm{ps}$ & $1.7 \mathrm{ps}$ & $2.4 \mathrm{ps}$ & $24 \mathrm{meV}$ \\
$\left(k_{\mathrm{S}}\right)^{-1}$ & $14.3 \mathrm{ps}$ & $18.2 \mathrm{ps}$ & $25.0 \mathrm{ps}$ & $40.0 \mathrm{ps}$ & $26 \mathrm{meV}$ \\
\hline
\end{tabular}

${ }^{a}$ Accounting for the $1 / T^{1 / 2}$ term in the pre-exponential factor of the Marcus equation, the activation energy for the BET process increases to $E_{\mathrm{A}}=36 \mathrm{meV}$.

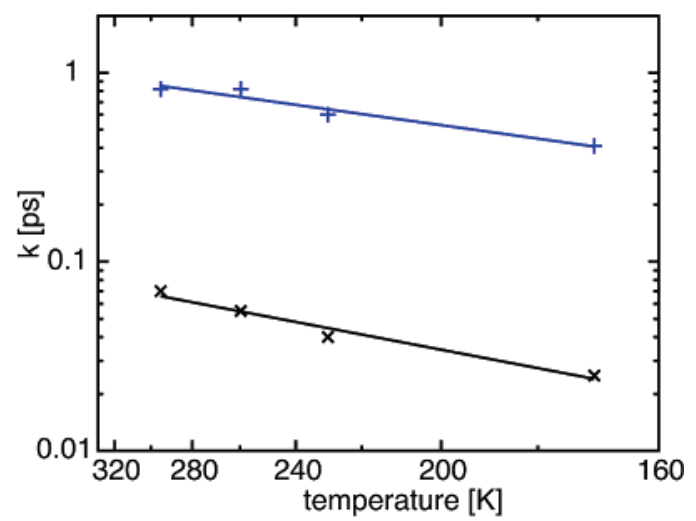

Figure 6. Arrhenius representation of the rate constants for ET (blue line) and spin relaxation (black line).

For a direct comparison of the simulation and the experimental results, the simulated time trace was convoluted with a Gaussian with a width of $150 \mathrm{fs}$, accounting for a delayed rise of the experiment signals due to the finite experimental response function and the forward ET reaction generating the RP. Furthermore, a small offset $(5 \%)$ was added to the simulated time traces, accounting for the signal contribution of free $\mathrm{Nb}^{+}$ molecules (cf. Experimental Section).

For each temperature, a two-dimensional array of simulated time traces was generated where in one dimension of the array the ET rate constant $k_{\mathrm{BET}}$ was varied in steps of $(0.1 \mathrm{ps})^{-1}$ and in the other dimension the spin relaxation time $\tau_{\mathrm{s}}$ was varied in steps of 0.5 ps. Best-fit values were obtained by comparing the zero field simulations with the experimental result obtained in the absence of an external field. The consistency of the obtained parameters was checked by comparing experimental and simulated $7 \mathrm{~T}$ time traces (Figure 4). The nice agreement between experiment and simulation shows that the model outlined above captures all features of the RP kinetics. This in turn renders the rate parameters of BET and spin relaxation derived from these simulations very reliably. Their values are summarized in Table 1. The temperature dependence of these parameters obeys Arrhenius behavior (Figure 6); the pertinent activation energies $E_{\mathrm{A}}$ are also compiled in Table 1. These numerical values will be addressed in the Discussion. We already stress here that the nearly equal activation energies for both processes are, to the best of our knowledge, just incidental and bear no deeper meaning.

\section{Discussion}

The experimental findings presented in this paper demonstrate once more that picosecond ET kinetics involving transition metal compounds can be influenced by spin processes. This can lead to multiexponential reaction kinetics. A careful analysis of these kinetics allows us to pin down genuine ET contributions and separate them from spin-induced processes. In this context, a 
magnetic field effect is an indispensable diagnostic tool. Once such an analysis is performed, the rate parameters extracted can be interpreted in the usual fashion. Here, these parameters are the ET rate constant and the spin relaxation time and their temperature dependence.

In a basic Marcus-type treatment, ${ }^{4,5}$ the ET rate constant $k_{\mathrm{BET}}$ in the non-adiabatic limit is cast in the following form:

$$
k_{\mathrm{BET}}=V^{2} \frac{4 \pi^{2}}{h \sqrt{4 \pi \lambda k_{\mathrm{B}} T}} \mathrm{e}^{-(\Delta G+\lambda)^{2} / 4 \lambda k_{B} T}
$$

where $\Delta G$ and $\lambda$ represent the free enthalpy change associated with the ET process and the total reorganization energy of the process, and $V$ stands for the electronic coupling. Qualitatively, the term $(\Delta G+\lambda)^{2} / 4 \lambda$ in the exponent can be equated with the small activation energy of $36 \mathrm{meV}$ obtained above; i.e., recombination has to be assigned to the activationless or inverted Marcus region. The precise relationship between the activation energy and the exponent is complex and is given in ref 5 . In the inverted region the classical Marcus treatment breaks down, and approaches incorporating quantum effects are in place if ET is coupled to high-frequency modes. These approaches, for instance the one put forth by Bixon and Jortner, ${ }^{7}$ predict a weak temperature dependence not only for the activationless $(|\Delta G|$ $=\lambda)$ but also for the inverted regime $(|\Delta G|>\lambda)$. Thus, based on the temperature dependence alone, the ET process cannot be classified as activationless or inverted. Supplementary electrochemical experiments addressing this issue are currently being undertaken. Assuming a close-to-activationless process, the Marcus treatment yields a coupling $V$ of $80 \mathrm{~cm}^{-1}$, which is in line with a non-adiabatic ET reaction. ${ }^{32} \mathrm{~A}$ comprehensive treatment of the ET kinetics of Fcp- $\mathrm{Nb}^{+}$, incorporating forward and backward ET, is the subject of ongoing research.

The data presented here represent-to the best of our knowledge - the first set of temperature-dependent picosecond electron spin relaxation data measured in the time domain. With a value of $\tau_{\mathrm{S}}=14.3 \mathrm{ps}$ at $295 \mathrm{~K}$, spin relaxation for the intermolecular $\mathrm{Fcp}-\mathrm{Nb}^{+}$system is well in line with values determined for isolated ferrocenes. The remaining deviation by a factor of $2-3\left(\tau_{\mathrm{S}}=6.6 \mathrm{ps}\right.$ measured with the present technique in the time domain for ethylferricenium ${ }^{15}$ and $\tau_{\mathrm{S}}=4.05 \mathrm{ps}$ measured by NMR methods on ferricenium ${ }^{33}$ ) will be addressed below.

The mechanisms of the relaxation of spin $1 / 2$ systems in solution can be divided into those driven by rotational diffusion and those independent of it. ${ }^{34}$ In detailed studies on the relaxation of ruthenium(III) complexes, Steiner et al. (see ref 35 and references therein) demonstrated that in this case the relaxation times $\tau_{\mathrm{S}}$, which are on the order of several picoseconds, are substantially shorter than rotational diffusion times. This is a clear indication that processes independent from rotational diffusion are operative. As early as 1939, van Vleck $^{36,37}$ suggested a mechanism not relying on rotational

(32) Newton, M D Chem. Rev. 1991, 91, 767

(33) Klingert, A Untersuchungen zur Elektronenspinrelaxation paramagnetischer Spin-1/2-Metallocene in flüssiger Lösung $\mathrm{PhD}$ thesis, Universität Konstanz, Konstanz, 2003

(34) Banci, L; Bertini, I ; Luchinat, C Nuclear and Electron Relaxation; VCH Verlagsgesellschaft: Weinheim, 1991

(35) Hötzer, K A ; Klingert, A ; Klumpp, T ; Krissinel, E ; Bürssner, D ; Steiner, U E J. Phys. Chem. A 2002, 106, 2207

(36) van Vleck, J H J. Chem. Phys. 1939, 7, 72

(37) van Vleck, J H Phys. Rev. 1940, 57, 426

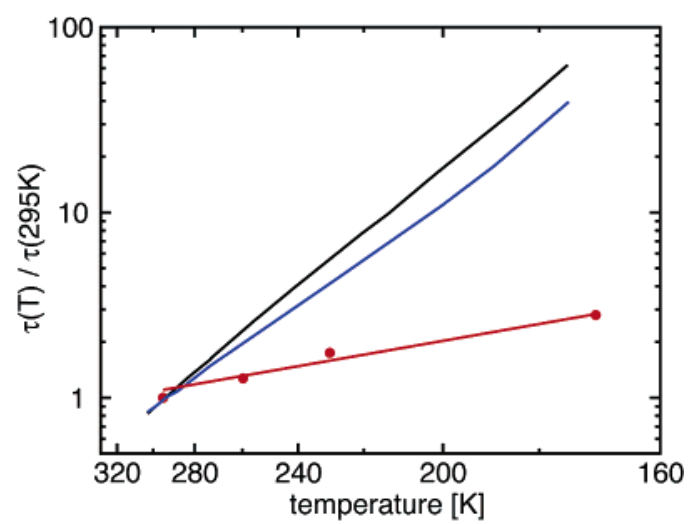

Figure 7. Temperature dependence of the spin relaxation time $\tau_{\mathrm{S}}$ (red line) and rotational correlation time $\tau_{\mathrm{c}}$ calculated from viscosity data for ethanol (black line) and methanol (blue line). Values are normalized to $T=295 \mathrm{~K}$.

motion. In his mechanism, the stochastic motion of solvent molecules generates fluctuating electric fields, which may induce "spin" transitions in highly spin-orbit coupled systems. In the 1960s, Finn and Orbach ${ }^{38,39}$ treated spin relaxation for systems with large spin-orbit coupling in solids as phonon-induced transitions between closely spaced electronic levels. This approach was then transferred to processes in solution by Kivelson, ${ }^{40}$ where incoherent solvent fluctuations take the role of phonons. According to this theory, spin $1 / 2$ systems with large spin-orbit couplings and electronically excited but thermally accessible states exhibit fast spin relaxation. For the ruthenium(III) complexes, Steiner et al. ${ }^{35}$ showed that spin relaxation times for these complexes are in line with the Orbach/Kivelson mechanism. For ferricenium, the same relaxation mechanism was put forth. ${ }^{15,22}$ However, since ferricenium is substantially smaller than ruthenium(III) complexes, the separation of the time scales between rotational motion and spin relaxation is not so distinct.

Based on the present results, a much clearer statement on the rotational independence of spin relaxation in ferricenium is possible. The rotational correlation time $\tau_{\mathrm{c}}$ of a molecule in solution is given by the Debye equation,

$$
\tau_{\mathrm{c}}(T)=V_{\mathrm{h}} \frac{\eta(T)}{k T}
$$

Regarding Fcp- $\mathrm{Nb}^{+}$as a rigid molecule with an approximate hydrodynamic volume $V_{\mathrm{h}}$ of ${ }^{4} / 3 \pi(7 \AA)^{3}$, one arrives at a roomtemperature correlation time $\tau_{\mathrm{c}} \approx 100 \mathrm{ps}$ for a viscosity of $\eta=$ $1 \mathrm{cP}$ (weighted mean of the viscosities of ethanol and methanol $\left.^{41}\right)$. This is much larger than the spin relaxation time $\tau_{\mathrm{s}}$ reported here. Since the ferricenium moiety might be able to rotate around the $\mathrm{C}-\mathrm{N}$ bond in $\mathrm{Fcp}-\mathrm{Nb}^{+}$, the effective hydrodynamic volume could be smaller, and the calculated correlation time $\tau_{\mathrm{c}}$ could come close to the measured spin relaxation time $\tau_{\mathrm{s}}$. When relying on the temperature dependence, no reference to this volume is required. In the temperature range covered by the experiments presented here, an increase of $\tau_{\mathrm{c}}$ by nearly 2 orders of magnitude is predicted by the Debye equation (Figure 7). In contrast, the experimental value of the spin relaxation time changes only by a factor of $\sim 3$.

(38) Finn, C B ; Orbach, R; Wolf, W P Proc. Phys. Soc. 1961, 77, 26

(39) Orbach, R Proc. Phys. Soc. 1961, 77, 821

(40) Kivelson, D J. Chem. Phys. 1966, 45, 1324

(41) Handbook of Chemistry and Physics; CRC Press Inc : Cleveland, OH, 1977 
These findings rule out rotational contributions being effective in the spin relaxation of ferriceniums. In the Orbach/Kivelson mechanism, fast spin relaxation independent of rotational processes is achieved through spin transitions via excited electronic states. Only in such a coupling scheme can purely electric interactions with the environment become effective. Due to Kramers' theorem, an electrostatic perturbation cannot induce direct transitions between the two components of the same Kramers doublet. The difference in energy between the two lowest Kramers doublets has to be supplied by vibrational and librational modes of the environment. Consequently, this process can be relevant only if these differences in energy are small enough that they can be in resonance with thermally accessible solvent modes. As shown experimentally by inelastic neutron scattering ${ }^{42}$ and underscored by DFT calculations, ${ }^{33}$ the Kramers doublets in ferricenium are separated by only a few tens of millielectronvolts, which is in good agreement with the activation barrier for this process.

Within this framework, spin relaxation rates should respond to changes of this separation. With increasing separation, the rates are expected to become slower. According to studies by Ammeter ${ }^{28}$ and Prins, ${ }^{29}$ the energetic separation of the two lowest Kramers doublets grows with increasing deviation from the axial symmetry of the ferricenium cation. Such a deviation is already present for ferricenium itself due to the constraints of the solvent environment, but it is much larger for the Fcp moiety due to the $\mathrm{C}_{3}$ bridge linking the two pentadienyl rings. The present finding of the spin relaxation rate for $\mathrm{Fcp}^{+\bullet}$ being by a factor of 2 slower than that for ferricenium is in line with this notion. An increased separation should also translate into a larger activation energy, $\Delta E_{\mathrm{s}}$. However, the value of $26 \mathrm{meV}$ reported here for $\mathrm{Fcp}^{+}$is even slightly lower than the one determined for ferricenium by NMR experiments (33-59 $\mathrm{meV}) .{ }^{33}$ At present we cannot judge whether this discrepancy really manifests shortcomings of the theoretical approach or is just a consequence of (small) different systematic errors of the two experimental techniques. Still, noting that both techniquesexperimentally disparate as they are-yield the same orders of magnitude for both the absolute relaxation rates and the activation barriers, rather small discrepancies should not be taken too seriously. Most important, the conclusion on the mechanism of the spin relaxation (Orbach-type mechanism) is strongly underscored by the present investigation.

\section{Conclusion}

Complex picosecond kinetics of an intramolecular radical pair bearing a ferricenium moiety is attributed to spin processes affecting its recombination dynamics. The assignment of the kinetic components rests on a quantitative analysis of magnetic field modulations and the temperature dependence of the picosecond time traces. This analysis led to electron transfer and spin relaxation rates and their respective activation parameters. The electron transfer is consistent with the activationless or inverted case according to Marcus theory. The activation energy of the spin relaxation points to an Orbach/Kivelsontype relaxation mechanism involving an excited Kramers doublet state. With respect to other electron transfer systems bearing a transition metal moiety, we would like to stress that, in the system presently studied, the spin effect influences only the

(42) Stebler, A ; Furrer, A ; Ammeter, J H Inorg. Chem. 1984, 23, 3493 kinetics of the system. Irrespective of the kinetics, the system recombines to the same ground state. This need not always be the case. For instance, singlet and triplet radical pair states might form different products. In this case, spin effects will have an impact on the reaction outcome, i.e., on reaction yields and the type of product formed.

\section{Experimental Section}

6.1. Chemicals and Solvents. The synthesis of the intramolecular ET system holding a ferrocenophane and a Nile blue moiety was described previously. ${ }^{25}$ The product was identified by NMR spectroscopy and CHN analysis with a purity of better than $99.5 \%$. Thin-film chromatography indicated a faint amount of a byproduct, possibly the $\mathrm{Nb}^{+}$dye. The ferrocenophane (Fcp) was synthesized according to ref 43 and characterized by NMR and CHN analysis. Nile blue $\left(\mathrm{Nb}^{+}\right)$ chloride was used as received from Sigma Aldrich.

Solvents used were of spectroscopic grade or better. A mixture of 1:2 (v:v) methanol:ethanol served as solvent with a low freezing point. ${ }^{44}$ Small amounts $(2 \% \mathrm{vol})$ of acetic acid were added to the solvents in order to prevent deprotonation of the exocyclic amino group of Fcp$\mathrm{Nb}^{+}$. Sample concentration was adjusted to an optical density of 1.0 for an optical path length of $1 \mathrm{~mm}$, corresponding to a concentration of $0.1 \mathrm{mM}$. Femtosecond experiments were performed in 1-mm fused silica cuvettes.

6.2. Experimental Techniques. Steady-State Spectra. Absorption spectra were recorded on either a Perkin-Elmer Lambda $2 \mathrm{~S}$ or a Varian Cary 100 Bio instrument. Fluorescence spectra were obtained by a Fluorolog-2 fluorimeter (Spex). The spectra reported were corrected for the spectral characteristics of the detection system.

Electron Paramagnetic Resonance. EPR spectra were recorded on a commercially available cw X-band spectrometer, ElexSys E500 (Bruker Biospin), operated at $9.42 \mathrm{GHz}$.

Magnetic Field-Dependent Transient Difference Absorption Experiments. The transient absorption experiments in high magnetic fields were performed using a femtosecond laser setup described in detail in refs 45 and 46. Magnetic fields of up to $9 \mathrm{~T}$ were supplied by a superconducting magnet (Oxford Instruments, SM-4000-9). Its incorporation in the transient absorption setup is described in refs 15 and 47. The relevant technical parameters of the experiment were the following. Pump pulses centered at $650 \mathrm{~nm}(150 \mathrm{fs}, 1 \mu \mathrm{J}, 1 \mathrm{kHz})$ were focused down to a diameter of $<500 \mu \mathrm{m}$ at the sample location, where they were crossed by a probe beam under a small angle of $\sim 5^{\circ}$. Probe pulses were obtained by selecting a spectral portion of a white light continuum using a grating monochromator. Care was taken to keep the diameter of the probe beam smaller than the one of the pump beam at the point of intersection. The difference absorption signal was obtained by blocking every second pump pulse; consecutive transmitted intensities of the probe beam after the sample in a "pump-on" and "pump-off" situation were detected at various time delays between the pump and probe pulses. The time resolution of the setup was $\sim 150$ fs. The relative polarization of pump and probe light was turned parallel to ensure best signal-to-noise ratio; in test experiments, no influences of rotational effects on the time traces could be detected. The high

(43) Rinehart, K L J ; Curby, R J J ; Gustafson, D H ; Harrison, K G ; Bozak, R E; Bublitz, D E J. Am. Chem. Soc. 1962, 84, 3263

(44) Ayotte, P ; Smith, R S ; Teeter, G; Dohnálek, Z; Kimmel, G A ; Kay, B D Phys. Rev. Lett. 2002, 88, 245505

(45) Lossau, H ; Kummer, A ; Heinecke, R ; Pöllinger-Dammer, F ; Kompa, $\mathrm{C}$; Bieser, G; Jonsson, T ; Silva, C M ; Yang, M M ; Youvan, D C; Michel-Beyerle, M E Chem. Phys. 1996, 213,1

(46) Pöllinger, F; Musewald, C; Heitele, H ; Michel-Beyerle, M E ; Anders, C ; Futscher, M ; Voit, G; Staab, H A Ber. Bunsen-Ges. Phys. Chem. Chem. Phys. 1996, 100, 2076

(47) Musewald, C ; Gilch, P; Hartwich, G ; Pöllinger-Dammer, F ; Scheer, H ; Michel-Beyerle, M E J. Am. Chem. Soc. 1999, 121, 8876 
magnetic fields employed here could potentially lead to signal distortions caused by, for instance, torques on mirror holders. To avoid such interferences, the use of ferromagnetic materials in a radius of $1.5 \mathrm{~m}$ around the center of the magnet was reduced to an absolute minimum. Furthermore, the optical setup was shielded with $\mu$-metal. To check for residual "technical" influences of the magnetic field, the transient absorption signal around time $t=0$ was recorded in zero and $7 \mathrm{~T}$ magnetic field. Theory predicts no magnetic field effect at time zero, and indeed the transient absorption signal was unaffected by the rise of the field.

Acknowledgment. Financial support by the Deutsche Forschungsgemeinschaft (MI 140/42-3) is gratefully acknowledged.

JA0547174 